\title{
Evaluating Storm Water Management Model Accuracy in Conditions of Mixed Flows
}

\author{
José G. Vasconcelos, Yasemin Eldayih, Yang Zhao and Jalil A. Jamily
}

\begin{abstract}
Stormwater systems, while designed to operate in free surface flow conditions, can undergo transition to pressurized flows during rapid filling conditions where inflows exceed the conveyance capacity of sewers and tunnels. Such conditions are referred to as mixed flows or flow regime transitions and pose a challenge to traditional unsteady numerical models. The Storm Water Management Model (SWMM) has an unsteady hydraulic solution algorithm based on an EXTRAN module that can simulate mixed flows. However, due to its link-node solution procedure, SWMM has limited ability to represent highly dynamic flow conditions involving the rapid filling of closed conduits and mixed flows. This work explores the accuracy of SWMM in representing three different experimental conditions involving mixed flows, testing the effects of spatial and temporal discretization on the results. The original recommendation presented in SWMM literature is revised and updated recommendations are proposed to improve SWMM simulation results in conditions involving mixed flows and rapid filling conditions.
\end{abstract}

\section{Introduction and Motivation}

Unsteady flows in stormwater systems are a complex, albeit prevalent, condition that is triggered by time-varying inflows that are admitted through several inlets or dropshafts present in these systems. In addition to these unsteady conditions, flows can also undergo a transition to pressurized flow regimes if inflows are rapidly admitted and exceed the conveyance capacity of the sewers and tunnels. This transition, referred to as mixed flow or flow regime transition (Song et al. 1983; Vasconcelos et al. 2006), is relevant because there are many operational problems linked to rapid filling conditions. These problems include, among others, excessive surging (Guo and Song 1990), structural damage (Zhou et al. 2002), stormwater geysering (Guo and Song 1991; Wright et al. 2011), and large air pocket entrapment (Vasconcelos et al. 2015). Thus, the ability to predict the occurrence of mixed flows caused by the rapid filling of stormwater systems is desirable.

Numerical simulation of mixed flows, often through one-dimensional models, is not straightforward since traditional unsteady flow solvers are not able to simulate both pressurized and free surface flow regimes. In general, mixed flow models are classified into two main groups, based on whether there are different sets of equations to simulate different regimes. Interface tracking models apply two sets of equations to simulate free surface and pressurized flow conditions, and require tracking the location of the pressurization front (Vasconcelos et al. 2006). Mixed flow models using a shock-capturing approach, on the other hand, use only the Saint-Venant equations to simulate both flow regimes and apply a conceptual model such as the Preissmann slot (Cunge and Wegner 1964) or the two-component pressure approach (Vasconcelos et al. 2006) to enable these equations to represent pressurized flows. A more detailed review of various mixed flow models is presented by Bousso et al. (2013).

One of the most popular models that applied to simulate flow conditions in stormwater systems is the Storm Water Management Model, SWMM (USEPA 2017). This model, currently in version 5.1 , is the result of a multi-decade effort and collaboration among various researchers, consultants and users, and it covers most relevant aspects of the hydraulics and hydrology of urban watersheds. One of the key modules of SWMM is its unsteady flow equation solver, which is based on the EXTRAN algorithm developed by Roesner et al. (1988) and Rossman (2006). This algorithm is based on a link-node solution, which does not use conduit discretization between two consecutive junctions. In this sense, SWMM can be classified as a type of interface-tracking mixed flow model since it uses two sets of equations to describe unsteady flows in closed conduits. When flows are in free surface mode, the Saint-Venant equations are solved (Rossman 2006):

$$
\begin{aligned}
& \frac{\partial A}{\partial t}+\frac{\partial Q}{\partial x}=0 \\
& \frac{\partial Q}{\partial t}+\frac{\partial\left(Q^{2} / A\right)}{\partial x}+g A \frac{\partial H}{\partial x}+g A\left(S_{f}-h_{L}\right)=0
\end{aligned}
$$

where:

$$
\begin{aligned}
A= & \text { cross-sectional area of the flow, } \\
Q= & \text { flow rate } \\
H= & \text { hydraulic head of water in the conduit (elevation } \\
& \text { head plus any possible pressure head), } \\
S_{f}= & \text { friction slope } \\
h_{L}= & \text { local energy loss per unit length of conduit, and }
\end{aligned}
$$

Vasconcelos, José G., Yasemin Eldayih, Yang Zhao and Jalil A. Jamily. 2018. Evaluating Storm Water Management Model Accuracy in Conditions of Mixed Flows. Journal of Water Management Modeling 27:C451. https://doi.org/10.14796/JWMM.C451

(c) CHI 2018. www.chijournal.org ISSN 2292-6062. 
$g=$ gravity acceleration .

The flow depth $y$ and $A$ are related via the geometry of cross sections, and can be calculated from $\mathrm{H}$. Friction slope is implemented in SWMM with the Manning equation.

EXTRAN applies another set of equations when a conduit flow is pressurized, which is the case when the junctions upstream and downstream from the conduit are pressurized:

$$
\begin{aligned}
& Q=A_{f} V \\
& \frac{d Q}{d t}=\left(\frac{g A_{f}}{L}\right)\left(\frac{\Delta H}{1+\Delta Q_{\text {friction }}+\Delta Q_{\text {losses }}}\right)
\end{aligned}
$$

where:

$A_{f}=$ full pipe flow area,

$V=$ average flow velocity in pressurized pipe,

$d t=$ routing time step size,

$L=$ conduit length,

$\Delta Q_{\text {friction }}=$ a nondimensional term that increases with the friction losses along the conduit, and

$\Delta Q_{\text {losses }}=$ a nondimensional term that increases with the local losses along the conduit.

It is implicitly assumed that pipe cross sectional elasticity and water compressibility are negligible, yielding a simple continuity equation.

More details of SWMM unsteady flow formulation, including the solution algorithm, are given in Rossman (2006). It is important to note that because of the link-node solution algorithm adopted by SWMM, there is a significant reduction of computational efforts to calculate unsteady flows. This can, on the other hand, impact the ability of SWMM to accurately represent rapid filling conditions and mixed flows, as shown in Ridgway and Kumpula (2008).

This is an important issue in the representation of rapidly filling sewers because pressure pulses in full pipe flow travel at much higher speeds (Wylie and Streeter 1993). Ridgway and Kumpula (2008) showed that spatial discretization and time step size decisively impact the ability of SWMM to simulate surging processes following closed conduit pressurization. In this interesting work, the authors improved the accuracy of experimental SWMM results given by Vasconcelos (2005). The effects of adding increasingly higher discretization to a $15 \mathrm{~m}$ long $94 \mathrm{~mm}$ diameter experimental apparatus were systematically evaluated. Four discretization strategies were used, involving 1, 32, 158 and 300 reaches to represent the pipe undergoing rapid filling. Time steps, which were in the order of milliseconds, were varied and the authors showed that for a certain spatial discretization size there was a determined time step value that improved modeling results against the experimental measurements. In particular, it was observed that either large or very small time steps would degrade the solution.

An appropriate choice of spatial and temporal discretization is very important to ensure the stability of numerical solutions for unsteady flows, as well as the quality of the solution. For the case of free-surface flow models that use spatial discretization and explicit numerical schemes, the Courant-FriedrichsLewy (CFL) condition determines whether a time step can cause instability (Toro 2001) according to the equation:

$$
\Delta t \leq \frac{C_{C F L} \Delta x}{S_{\max }}
$$

where:

$$
\begin{aligned}
\Delta x= & \text { size of the spatial discretization (i.e. pipe reach), } \\
C_{C F L}= & C F L \text { coefficient (also known as the Courant } \\
& \text { number) of the computation, and } \\
S_{\text {max }}= & \text { celerity of the pressure waves propagating in the } \\
& \text { solution domain. }
\end{aligned}
$$

Equation 5 is valid for both free surface and pressurized flows, but in pressurized flows the celerity of pressure waves is much larger. In the case of explicit numerical schemes, the maximum admissible value of $C_{C F L}$ in most numerical schemes is 1. For open channel flows, the value of $S_{\text {max }}$ is a function of local flow velocity $V$, cross sectional area of the flow $A$ and its free surface width $B$ :

$$
S_{\max }=\max \left(\sqrt{\frac{g A}{B}}+V\right)
$$

With regards to the choice of time steps, there is a consensus among SWMM modelers that the size should be small enough to capture the unsteady nature of flows in a simulation. As a result, it is common to encounter flow routing time steps in the order of few seconds for various SWMM applications involving unsteady flows (Rossman 2006; Gironas et al. 2009). Also, SWMM has a feature that variable time steps can decrease their values automatically when EXTRAN detects sudden changes in flow conditions. A recommendation for time step size provided by Roesner et al. (1988) in terms of calculation stability is:

$$
\Delta t_{\text {EXTRAN }}=\min \left(\frac{L}{\sqrt{g D}}\right)
$$

where:

$$
\begin{aligned}
\Delta t_{\text {EXTRAN }}= & \text { upper limit of routing time step recommended in } \\
& \text { the EXTRAN documentation, and } \\
D= & \text { conduit diameter. }
\end{aligned}
$$

The condition must be observed for all conduits in the project, hence the time step will be determined by the one conduit with the smallest value of $L /(g D)^{0.5}$. The recommended stability criteria for SWMM models are mostly dependent on conduit geometry characteristics, which are constant over a simulation. In contrast, free surface flow models that use explicit schemes and discretization adopt time steps that depend both on geometric characteristics and on time varying flow conditions. A final remark regarding time steps in models is that while large time steps can lead to computational instability, small time steps can degrade the solution due to low Courant number values (Toro 2001). 
To date there are no specific guidelines on setting time steps for cases involving mixed flow conditions in SWMM. While the work by Ridgway and Kumpula (2008) showed that discretization via dummy links in SWMM can improve the SWMM ability to simulate surges following the rapid filling of conduits, some open questions still exist. For instance, only a single experimental dataset was evaluated by Ridgway and Kumpula (2008), and other relevant flow conditions involving mixed flows should also be evaluated. Another question is whether a general recommendation for spatial and temporal discretization for SWMM modeling can be proposed in conditions involving mixed flows. These questions are the focus of the present work.

\section{Objectives}

This work aims to evaluate the capability of the SWMM unsteady flow algorithm EXTRAN to represent flow conditions that involve mixed flows. The goal is achieved by assessing the ability of SWMM to represent pressures and velocities obtained in conditions representing mixed flows in previously reported in three separate experimental investigations. The following Methodology section presents the methodology of the study, including modeling assumptions, conditions and discretization strategy. A comparison between SWMM modeling and experimental results is given in the Results and Discussion section, followed by a Conclusions section, where future work is also recommended.

\section{Methodology}

\subsection{Selected Experimental Investigations}

Three different experimental studies were selected to assess the ability of SWMM to represent conditions involving rapid filling, and the choice was motivated by the different conditions that are represented in these studies. The three studies, which are presented in the schematics in Figure 1, are:

1. Vasconcelos (2005): Study of surging in a downstream shaft that followed the rapid filling of a horizontal pipe, with an inflow at the upstream end and surge shaft at the downstream end.

2. Trajkovic et al. (1999): Presents different cases involving the formation of pipe filling bores by blocking outflows through the operation of a downstream valve, and partial reopening leading to a partial pipe depressurization.

3. Aureli et al. (2015): Presents a case in which a pipeline has an intermediate low point, and with an intermediate knife gate valve. The valve separates a pipe segment with pressurized flow from an empty segment, and is suddenly opened which leads to a sloshing process.

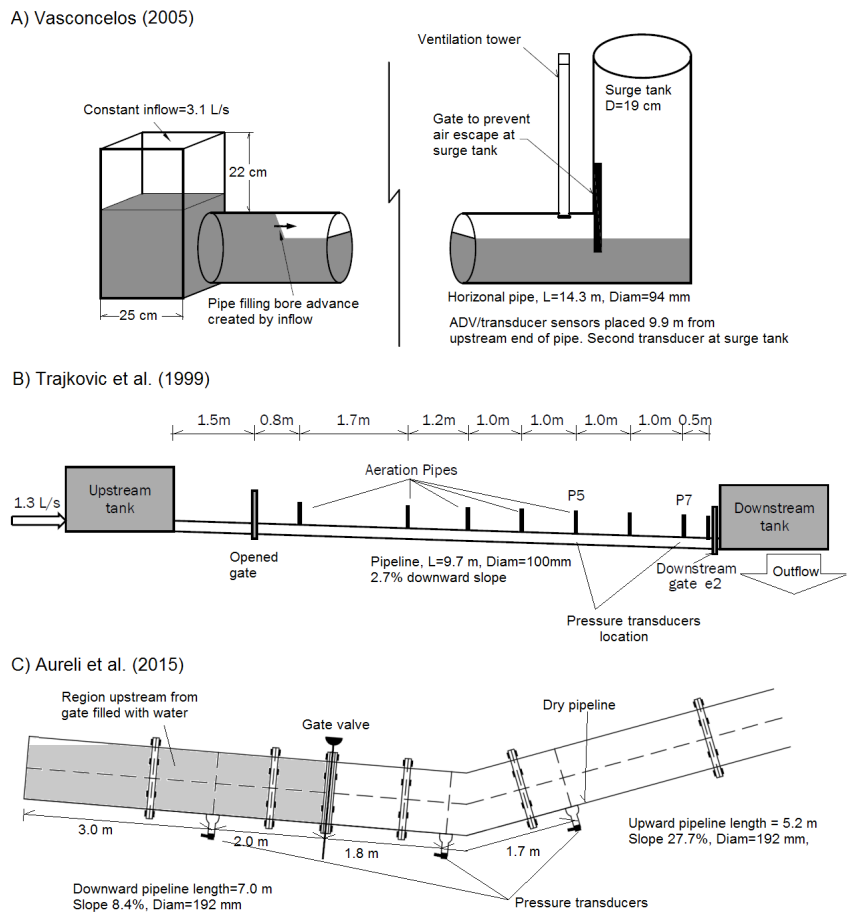

Figure 1 Sketches of apparatuses considered in Vasconcelos (2005), Trajkovic et al. (1999) and Aureli et al. (2015).

These flow conditions are all highly dynamic, and relevant to the goal of assessing the ability of SWMM to represent mixed flow conditions. All these investigations used plexiglass pipes, and the length of the apparatus ranged from $10 \mathrm{~m}$ to $15 \mathrm{~m}$, while the diameters ranged from $94 \mathrm{~mm}$ to $192 \mathrm{~mm}$. Clearly, all these dimensions are much smaller than actual drainage systems, which will affect the selection of time steps used in the assessment. In these tests, both pressure measurements and velocity measurements were promoted at selected locations.

The experimental study in Vasconcelos (2005) consisted of an inflow tank and a surge tank linked by a $14.3 \mathrm{~m}$ long $94 \mathrm{~mm}$ diameter horizontal pipe, wherein water was in a quiescent condition. The downstream shaft had an overflow elevation much greater than the value at the upstream inflow tank. A sudden inflow admission at the upstream end created a pipe filling bore that propagated downstream and led to the development of inertial oscillations in the surge tank upon the bore arrival there. While oscillations existed in the surge tank, overflows occurred in the upstream inflow tank. A ventilation tower was present at the downstream end and avoided air pressurization during the filling process.

The representation of the boundary conditions in Trajkovic et al. (1999) involved the addition of a steady inflow rate of $1.3 \mathrm{~L} / \mathrm{s}$ at an upstream junction, which represented an upstream tank. The apparatus was $\sim 10 \mathrm{~m}$ long, with a $10 \mathrm{~cm}$ diameter pipe with acrylic walls. A free outfall existed in the downstream end of the 2.7\% slope pipeline, which flowed in supercritical mode until a pipe filling bore was created by the rapid closure of a valve at that location. 
Finally, the boundary conditions in Aureli et al. (2015) were represented by regular junctions, with an outlet placed at the downstream end. The initial $7.0 \mathrm{~m}$ of the pipeline had an $8.4 \%$ downward slope, followed by a $5 \mathrm{~m}$ long segment with an upward slope of $27.7 \%$, with an internal gate valve located $5 \mathrm{~m}$ from the upstream end. The diameter of the acrylic pipe was $0.192 \mathrm{~m}$. Water in a quiescent condition was present upstream of the valve, and sloshing was initiated by sudden valve opening.

\subsection{SWMM Modeling Setup and Assessment}

The development of the SWMM models involved the creation of intermediate junctions (also referred to herein as nodes) between the natural boundaries of the model as a means of introducing discretization. As a result, for each one of the three experimental studies, SWMM models with varying numbers of reaches were created. The number of reaches for every experimental study consisted of four values that ranged from 10 reaches up to 120 reaches. Instead of the default minimum nodal surface area used in SWMM $\left(1.14 \mathrm{~m}^{2}\right)$, much smaller junction areas, ranging from $10^{-3} \mathrm{~m}^{2}$ to $10^{-6} \mathrm{~m}^{2}$, were used. This step was taken so that the hydraulic storage provided by junctions with larger areas did not influence the results of hydraulic calculations.

Pipe wall roughness values used in the SWMM modeling were consistent with the values reported in the experimental studies, ranging from 0.008 to 0.012 . All calculations were performed using the dynamic wave equation, which is equivalent to using Equations 1 through 4, keeping the inertial terms and using a small head convergence tolerance in the order of $\leq 10^{-6} \mathrm{~m}$. Because one objective of this study was to study the effect of time steps on modeling accuracy, the option of variable time steps was disabled, and four values of routing time step were used, varying from $1 \mathrm{~s}$ down to $10^{-3} \mathrm{~s}$. The reporting time step was the smallest possible value using the SWMM5 GUI, equal to $1 \mathrm{~s}$. Simulation times matched the period in which data collection lasted in experiments, usually in the range $1 \mathrm{~min}$ to $2 \mathrm{~min}$. A detailed discussion on the flow conditions in each experiment is given below in the results and discussion session.

Regarding boundary conditions representation for the Vasconcelos (2005) study, a storage unit was placed in each of the upstream and downstream ends of the SWMM model, with fixed plan areas that correspond to those reported in the experimental study. The horizontal pipeline had a constant water depth of 73 $\mathrm{mm}$ in a $94 \mathrm{~mm}$ diameter pipe, in a quiescent condition. A fixed inflow of $3.1 \mathrm{~L} / \mathrm{s}$ was suddenly admitted to the upstream storage unit, thereby creating the moving bore. Overflows thus occurred only at the upstream end, and these were collected by a rectangular weir placed at that location, which was then connected to the SWMM model free outfall.

The partial reopening of the Trajkovic et al. (1999) study was represented in SWMM by opening a parallel pipe that connected the last junction to the SWMM model free outfall via control rules. This parallel pipe had rectangular cross section with the area matching the opened area of the valve. An orifice could also be used to represent the valve partial reopening, but it was observed in the simulations that the time response of an orifice opening was not as instantaneous as the corresponding maneuver of a pipe. Finally, the representation of the boundary conditions of Aureli et al. (2015) was modeled in SWMM via a control rule that set the status of the conduit downstream of the valve to be open.

Each experimental modeling study was simulated using multiple discretizations and time steps, with a total of 16 SWMM models developed, and 48 total conditions represented. These conditions are shown in Table 1, along with the time step calculated by Equation 8.

Table 1 Conditions simulated in SWMM to represent each experimental study.

\begin{tabular}{ccccc}
\hline \multirow{2}{*}{ Investigation } & $\begin{array}{c}\text { No. of interm. } \\
\text { junctions used }\end{array}$ & $\begin{array}{c}\text { Average spatial } \\
\text { discretization (m) }\end{array}$ & $\begin{array}{c}\text { Calculated with } \\
\text { Eq. } 7(\mathrm{~s})\end{array}$ & $\begin{array}{c}\text { Time step sizes } \\
\text { used (s) }\end{array}$ \\
\hline Vasconcelos (2005) & 10 & 1.43 & 1.49 & \\
& 20 & 0.715 & 0.74 & \\
& 50 & 0.286 & 0.30 & 1.000 \\
Trajkovic et al. (1999) & 100 & 0.143 & 0.15 & \\
& 10 & 1.0 & 1.01 & 0.100 \\
& 20 & 0.5 & 0.50 & \\
& 50 & 0.2 & 0.20 & 0.010 \\
Aureli et al. (2015) & 100 & 0.1 & 0.10 & \\
& 12 & 1.0 & 0.73 & \\
& 24 & 0.5 & 0.001 \\
& 60 & 0.2 & 0.15 & \\
& 120 & 0.1 & 0.07 & \\
\hline
\end{tabular}

The metric used to assess the accuracy of the modeling work was the $L_{1}$ norm. It was adopted to compare the discrepancies between the simulation results for pressure and velocity at selected locations and the corresponding experimental measurements. The $L_{1}$ norm is calculated by:

$$
L_{1}=\sum_{i=1}^{N_{j}}\left|X_{\text {exp }}-X_{m o d e l}\right|
$$

where:

$$
\begin{aligned}
N_{j} & =\text { number of junctions in the SWMM model, } \\
X & =\text { pressure or velocity values, and } \\
= & \text { experimental values and modeled values, } \\
\text { exp, model } & \text { respectively. }
\end{aligned}
$$

We reiterate that the time steps used in this work are much smaller than in typical SWMM models. This is due to the small dimensions of the conduits used in the experimental tests.

\section{Results and Discussion}

\subsection{Vasconcelos (2005) Comparison}

The SWMM results are compared with measured velocities in an intermediate section of the pipe and the water level in the surge tank. For this analysis, all results obtained with $1 \mathrm{~s}$ time step were so unstable that they are not included. This finding is relevant since this time step is in the range of the calculated results from Equation 7 for 10 to 12 intermediate junctions, as shown in Table 1. 
A comparison between experimental measurements of velocity at coordinate $9.9 \mathrm{~m}$ and the simulation results obtained with SWMM is presented in Figure 2. In order to facilitate the comparison, results are grouped by the time step size, from $0.1 \mathrm{~s}$ down to $0.001 \mathrm{~s}$. Again, results with a $1 \mathrm{~s}$ time step are not shown because they were too unstable and inaccurate. In general, results in Figure 2 indicate that simulated results with larger numbers of intermediate junctions (50 and 100) yield poorer results with the larger time step of $0.1 \mathrm{~s}$. With the smallest time step $(0.001 \mathrm{~s})$, the situation is reversed, showing a worse agreement with models that use coarser discretization (i.e. fewer junctions).

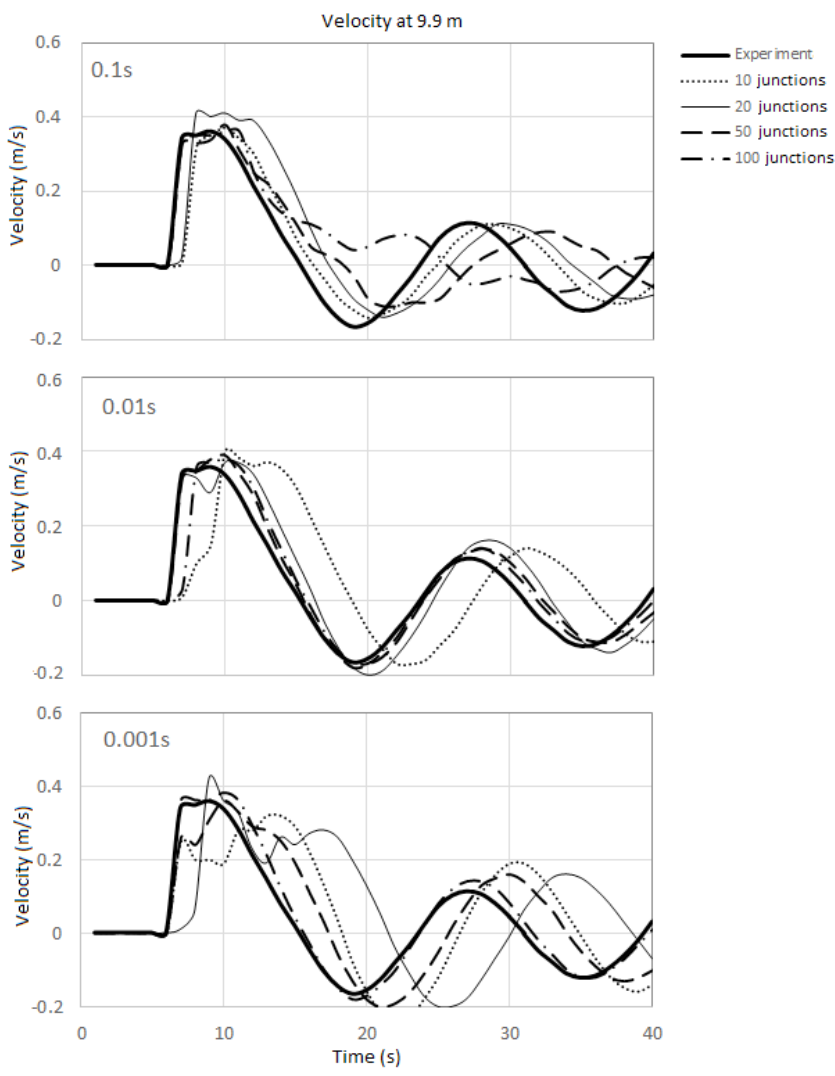

Figure 2 Experimental (Vasconcelos 2005) and simulated results of velocity at $9.9 \mathrm{~m}$ station using SWMM.

After the SWMM simulation, results are compared with the experimental measurements and the values of $L_{1}$ norms are calculated, as shown in Table 2. $L_{1}$ norm results indicate that the best agreements are attained with $\Delta T=0.01 \mathrm{~s}$ for 50 and 100 intermediate junctions; when $\Delta T=0.001 \mathrm{~s}$ the best agreement is attained with 100 junctions.

Table $2 L_{1}$ norm for velocity results at $9.9 \mathrm{~m}$ (Vasconcelos 2005).

\begin{tabular}{ccccc}
\hline$\Delta T(\mathrm{~s})$ & 10 junctions & 20 junctions & 50 junctions & 100 junctions \\
\hline 0.1 & 3.32 & 1.93 & 2.97 & 2.96 \\
0.01 & 5.16 & 2.02 & 0.97 & 1.13 \\
0.001 & 6.36 & 4.69 & 3.14 & 0.73 \\
\hline
\end{tabular}

These general observations are also valid for the comparison between experimental and modeled results of the surge tank water level, shown in Figure 3 , and in the $L_{1}$ norm results presented in Table 3. While simulation results obtained with coarser discretization are qualitatively well represented among all time steps, results with 50 and 100 junctions are not accurate with $\Delta T=0.1 \mathrm{~s}$. However, $L_{1}$ norm results indicate that the most accurate results are obtained with the models constructed with 100 junctions, using $\Delta T$ values of $0.01 \mathrm{~s}$ and $0.001 \mathrm{~s}$.

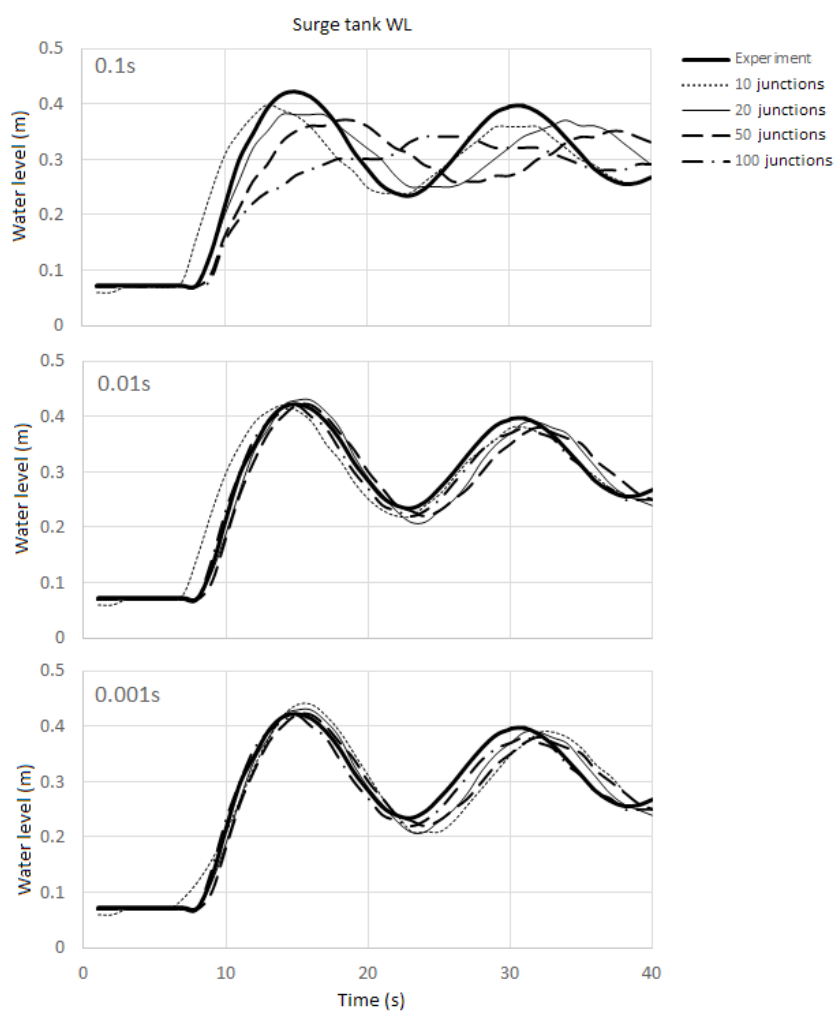

Figure 3 Experimental (Vasconcelos 2005) and simulated results of water level at the surge tank $14.3 \mathrm{~m}$ using SWMM

Table $3 L_{1}$ norm for surge tank WL at $9.9 \mathrm{~m}$ (Vasconcelos 2005).

\begin{tabular}{ccccc}
\hline$\Delta T(s)$ & 10 junctions & 20 junctions & 50 junctions & 100 junctions \\
\hline 0.1 & 1.08 & 1.29 & 2.20 & 2.12 \\
0.01 & 1.29 & 0.65 & 0.82 & 0.42 \\
0.001 & 2.54 & 1.55 & 1.41 & 0.46 \\
\hline
\end{tabular}

\subsection{Trajkovic et al. (1999) Comparison}

One important distinction of the experimental conditions considered in Trajkovic et al. (1999) is the existence of supercritical flows prior to the operation of the downstream valve and created a backward moving bore. This condition was modeled in SWMM by setting the status of the conduit at the downstream end to closed via control rules. After $30 \mathrm{~s}$ from the valve closure, the partial reopening of $0.028 \mathrm{~m}$ of the valve in the Trajkovic et al. (1999) experiments resulted in a partial depressurization of the apparatus. 
The results presented below, which involved the use of a $2.7 \%$ slope, created some regularly spaced oscillations in the SWMM flow depth prior to any valve operation. By increasing the size of the junction plan area to $0.001 \mathrm{~m}^{2}$ these oscillations decreased to a point that the model was stable. The causes of these oscillations are not clear, but it is speculated that they may be linked to the occurrence of supercritical flows along with various intermediate junctions. It was anticipated that results of flow regime transition upon valve operation would be more complex to interpret. As in the case of the results of Vasconcelos (2005), $1 \mathrm{~s}$ time step results were too unstable and are not presented.

Indeed, as is shown in Figure 4, there was no clear trend regarding the agreement between SWMM results and corresponding pressure measurements at the $7 \mathrm{~m}$ station, $0.5 \mathrm{~m}$ away from the gate. It may be noticed that, in general, all modeling results correctly predicted the pressure increase and eventual pressurization as the gate was closed at $T=0 \mathrm{~s}$, and the pressure drop upon the partial gate reopening at $T=0 \mathrm{~s}$. However, erratic pressure oscillations are also seen. Results with the largest time step ( $\Delta T=0.1 \mathrm{~s}$ ) presented the smallest oscillations in SWMM pressure results. Stronger oscillations are observed with smaller time steps, irrespective of the number of junctions used in the simulations.
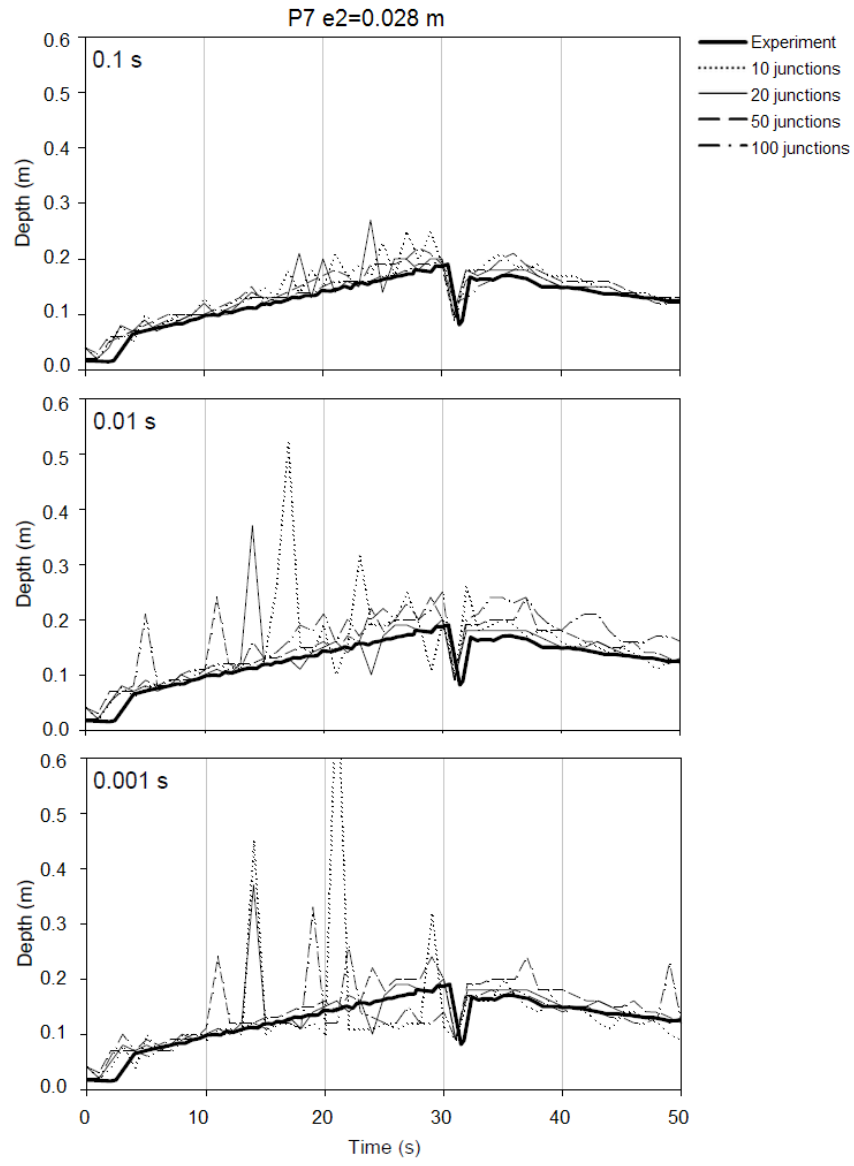

Figure 4 Experimental results by Trajkovic et al (1999) and simulated results of water pressure head at P7 using SWMM.
The effects of the erratic oscillations are noticed in the calculated $L_{1}$ norms, shown in Table 4 . As can be seen, the best results were obtained with the smallest time step used in computation. There is no trend regarding the number of junctions and the value of $L_{1}$ for the smallest time steps. Other alternatives that could help improve the stability of supercritical flow simulations in SWMM, such as damping the inertial terms, were not considered, in order to maintain the consistency of the analysis among the three different experimental setups used in the simulation. However, this should be addressed in future investigations.

Table $4 L_{1}$ norm for pressure head results at P7 (Trajkovic et al. 1999).

\begin{tabular}{ccccc}
\hline$\Delta T(s)$ & 10 junctions & 20 junctions & 50 junctions & 100 junctions \\
\hline 0.1 & 0.77 & 1.11 & 0.89 & 0.36 \\
0.01 & 0.80 & 1.65 & 1.15 & 1.93 \\
0.001 & 0.80 & 2.15 & 1.15 & 1.17 \\
\hline
\end{tabular}

\subsection{Aureli et al. (2015) Comparison}

The experimental results presented by Aureli et al. (2015) are very interesting since they not only create conditions of flow regime transition, but also oscillatory flows. Upon the rapid opening of the gate valve, flow propagates toward the empty portion of the apparatus, and, over time, the boundaries of the apparatus show cycles of wetting and drying with velocity reversion. It was anticipated that this would be a challenging test for SWMM modeling. Unlike the other comparisons in this work, the SWMM models were constructed with 12, 24, 60 and 120 intermediate junctions.

A comparison between SWMM results and experimental measurements of the velocity at $X=6.74 \mathrm{~m}$ (near to the apparatus bottom) is shown in Figure 4, except for the case when the time step was $1 \mathrm{~s}$. Results indicate that for the largest time steps the models with largest number of junctions yielded the worst results. When $\Delta T$ was set to $0.1 \mathrm{~s}$, the frequency of oscillations and the magnitude of velocity were altered for the model with 120 intermediate junctions. On the other hand, velocity magnitudes yielded by SWMM models with 12 and 24 junctions were underpredicted when $\Delta T=0.001 \mathrm{~s}$. As Table 5 shows, $L_{1}$ norm results indicate that the best results were obtained with $\Delta T=0.001 \mathrm{~s}$ and with 60 and 120 intermediate junctions.

Table $5 L_{1}$ norm for velocity at $X=6.74 \mathrm{~m}$ (Aureli et al. 2015).

\begin{tabular}{ccccc}
\hline$\Delta T(\mathrm{~s})$ & 10 junctions & 20 junctions & 50 junctions & 100 junctions \\
\hline 0.1 & 9.04 & 12.58 & 14.68 & 27.82 \\
0.01 & 8.54 & 8.65 & 7.47 & 8.44 \\
0.001 & 9.44 & 11.8 & 6.88 & 6.73 \\
\hline
\end{tabular}

A comparison of the pressure head at $X=8.52 \mathrm{~m}$ obtained in the Aureli et al. (2015) experiments and corresponding SWMM5 simulations is shown in Figure 5 , with $L_{1}$ norm 
calculation results given in Table 6. This location is relevant since the flow periodically reaches that location during the experiments and recedes from it. Similar to the velocity comparison, the worst SWMM results were for 120 junctions and the largest time step of $\Delta T=0.1 \mathrm{~s}$, whereas results obtained with the 12 and 24 junctions were comparatively better. In general, the best results were found for $\Delta T=0.01 \mathrm{~s}$, with the period of the oscillations most closely matching the experimental results. Results of the smallest $\Delta T=0.001 \mathrm{~s}$ with the larger number of junctions were fairly accurate, but the pressure head values were greatly underpredicted for the models with 12 and 24 junctions.

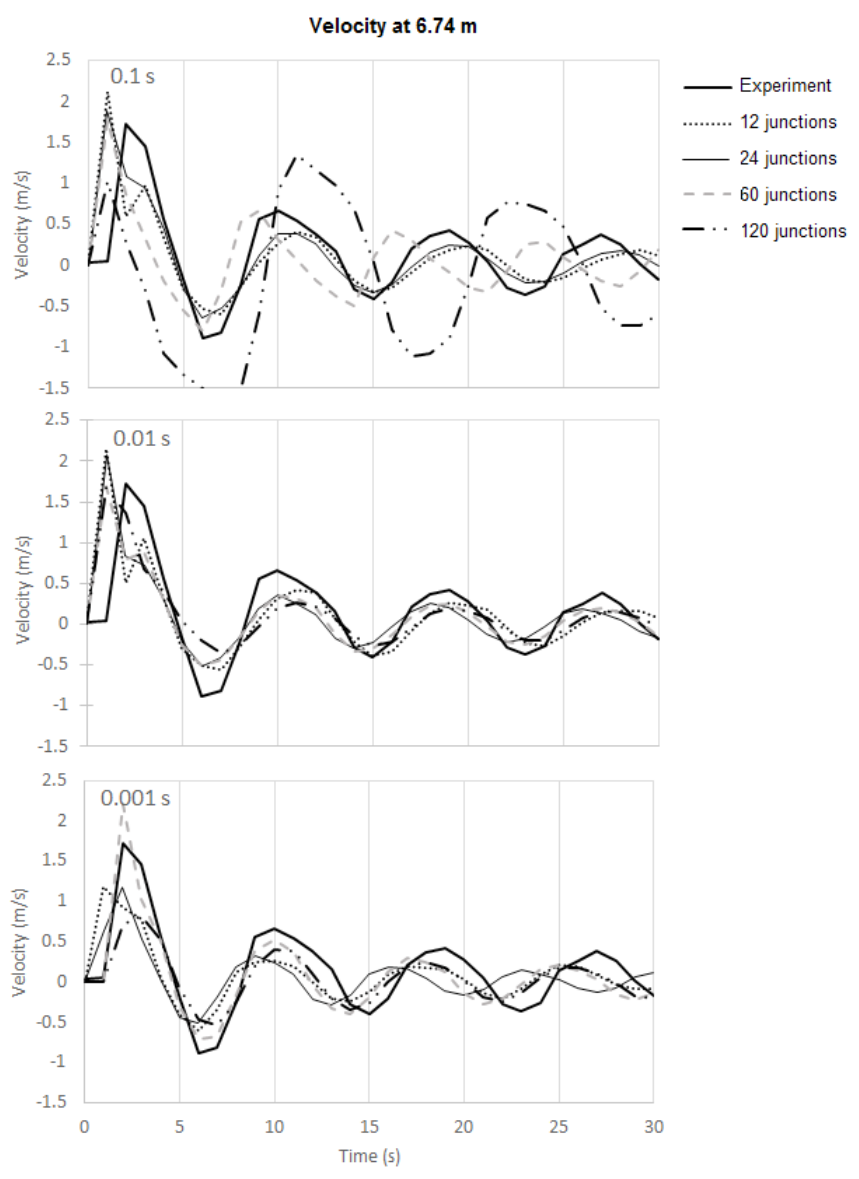

Figure 5 Experimental results (Aureli et al. 2015) and simulated results of water velocity at $X=6.74 \mathrm{~m}$ using SWMM.

Table $6 L_{1}$ norm for pressure head at $X=8.52 \mathrm{~m}$ (Aureli et al. 2015).

\begin{tabular}{ccccc}
\hline$\Delta T(\mathrm{~s})$ & 10 junctions & 20 junctions & 50 junctions & 100 junctions \\
\hline 0.1 & 0.88 & 0.97 & 10.06 & 17.67 \\
0.01 & 0.85 & 0.82 & 0.81 & 1.03 \\
0.001 & 1.41 & 1.50 & 1.18 & 0.86 \\
\hline
\end{tabular}

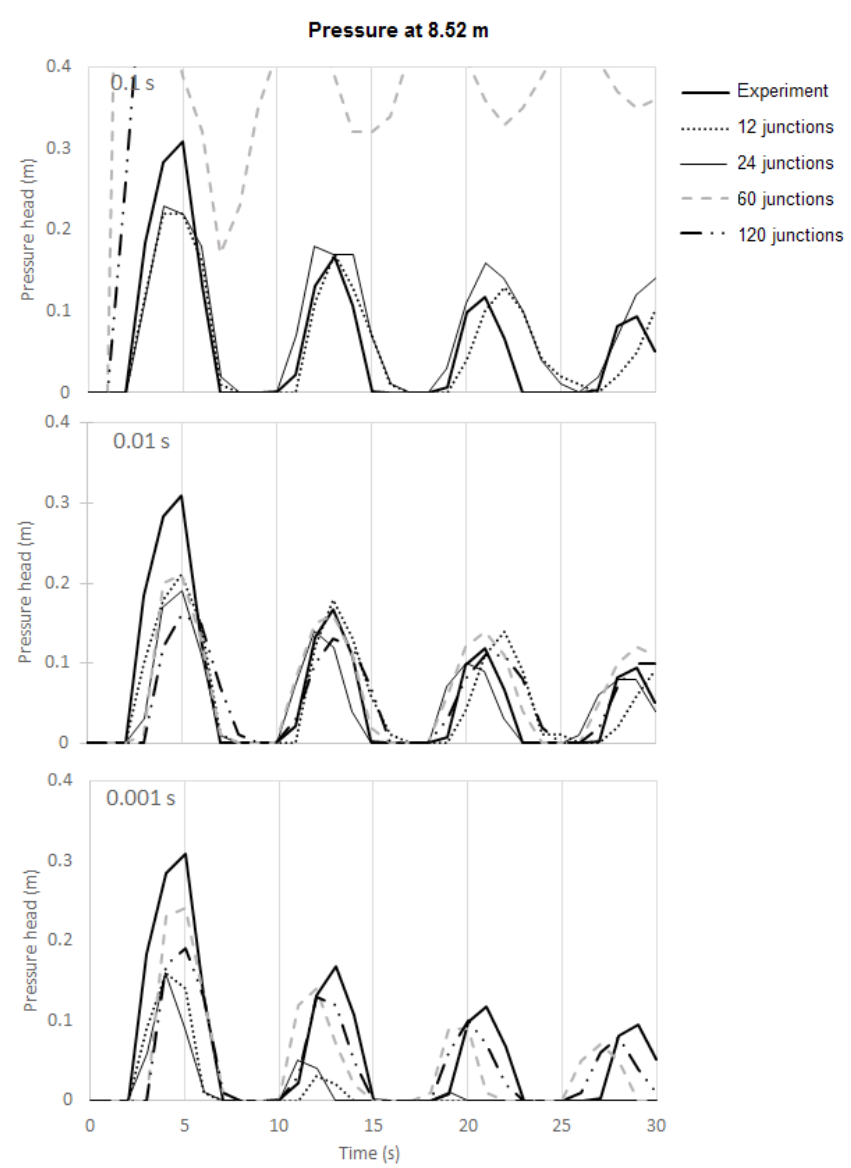

Figure 6 Experimental results (Aureli et al. 2015) and simulated results for the pressure head at $8.52 \mathrm{~m}$ using SWMM.

\subsection{Alternative to Improve Simulation Results Involving Flow Regime Transition}

The simulation results for all experimental conditions indicate that the combination of time step and number of intermediate junctions affect model accuracy. In general, a combination of more intermediate junctions (i.e. more spatial discretization) with larger time steps causes a decrease in modeling accuracy. Likewise, fewer intermediate junctions and smaller time steps negatively influence the accuracy of models. Both results are expected from a theoretical standpoint, since such extreme cases are associated with Courant numbers that deviate most from unity. Solutions yielded by linear numerical schemes and small Courant numbers are impacted either by diffusivity or dispersion (Toro 2001). On the other hand, Courant numbers that greatly exceed 1.0 will ignore fast-occurring transient features in open channel flow, which is a key feature of flows that involve flow regime transition.

Adjustment of time steps in SWMM based on the Courant number, which in turn is based on flow velocity and celerity, is not possible at present. On the other hand, the time step to maintain computation stability recommended by Roesner et al. (1988) is 
easily attainable. Yet the simulations involving transition into pressurized flow regimes given here indicate that yields poor results. A small modification of Roesner et al. (1988) is proposed, intended to reduce this time step size:

$$
\Delta t_{\text {mod }}=0.1 \frac{\Delta x}{\sqrt{g D}}
$$

where is the proposed time step to perform simulations involving a transition from free surface into pressurized flow conditions. The time step is $10 \%$ of the value proposed by Roesner et al. (1988) to ensure stability; other values that are in the same order of magnitude for the reducing factor, from $20 \%$ to $5 \%$, were also tested with comparable results, albeit not as systematically. All simulations with all discretization levels were repeated with the time step calculated according to Equation 9, and the results are shown in Figures 7, 8 and 9. The resulting time steps were in the range $0.1 \mathrm{~s}$ to $0.01 \mathrm{~s}$ for the cases considered.

As can be seen from the results using time steps calculated by Equation 9, there is, in general, good agreement between SWMM modeled results and experimental measurements, regardless of the number of intermediate junctions used in the simulation. It can be seen that the results with the smallest number of intermediate junctions yield some overestimation of the pressure-velocity oscillation period. Results obtained with larger numbers of intermediate junctions (50 to 120) did not dramatically improve the agreement between simulated results and experimental measurements. Thus it seems that when Equation 9 is used the recommended alternative is to adopt a range of 20-24 intermediate junctions for conduits with L/D around 100.
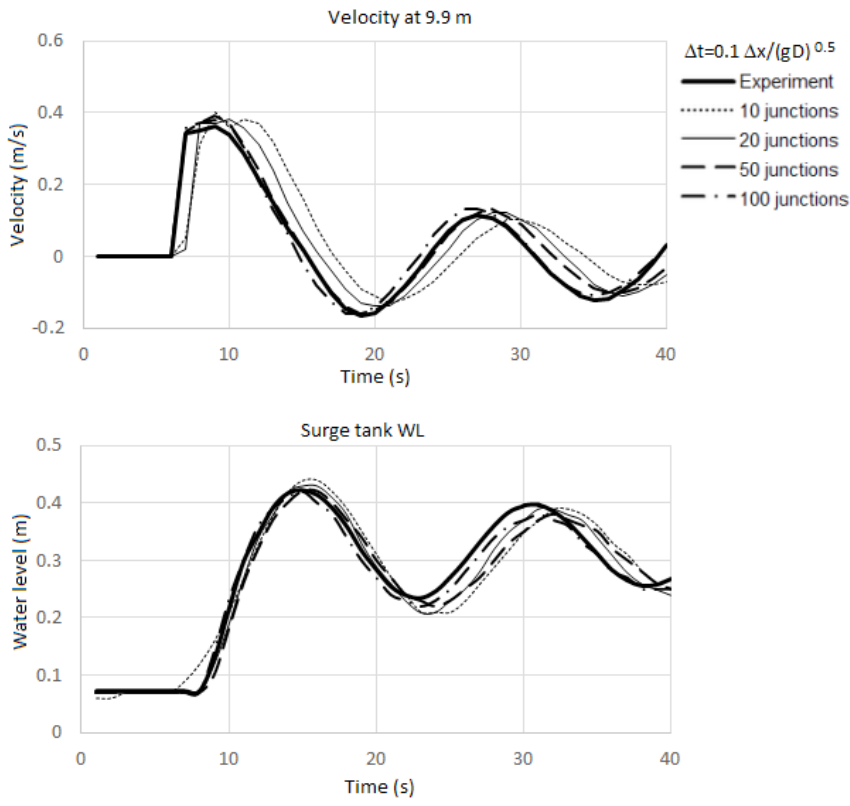

Figure 7 Experimental results (Vasconcelos 2005) and simulated results of velocity at $9.9 \mathrm{~m}$ station and water level in the surge tank using SWMM and time steps calculated by Equation 9.

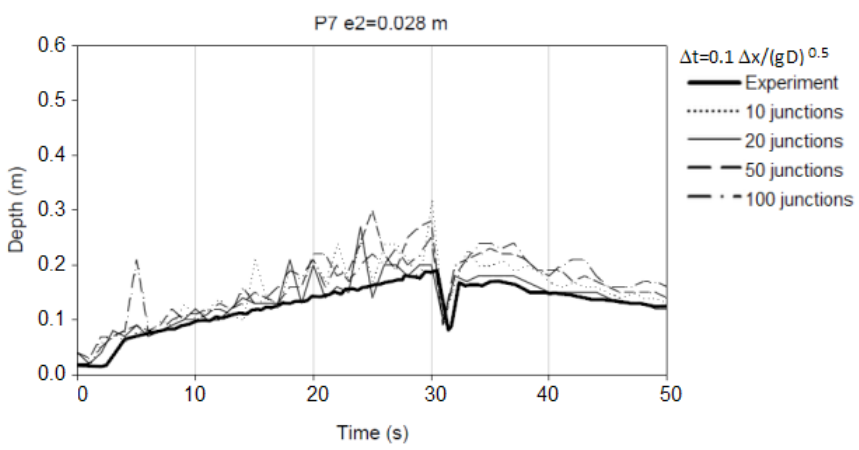

Figure 8 Experimental results (Trajkovic et al. 1999) and simulated results of water pressure head at P7 using SWMM and time step calculated by Equation 9.
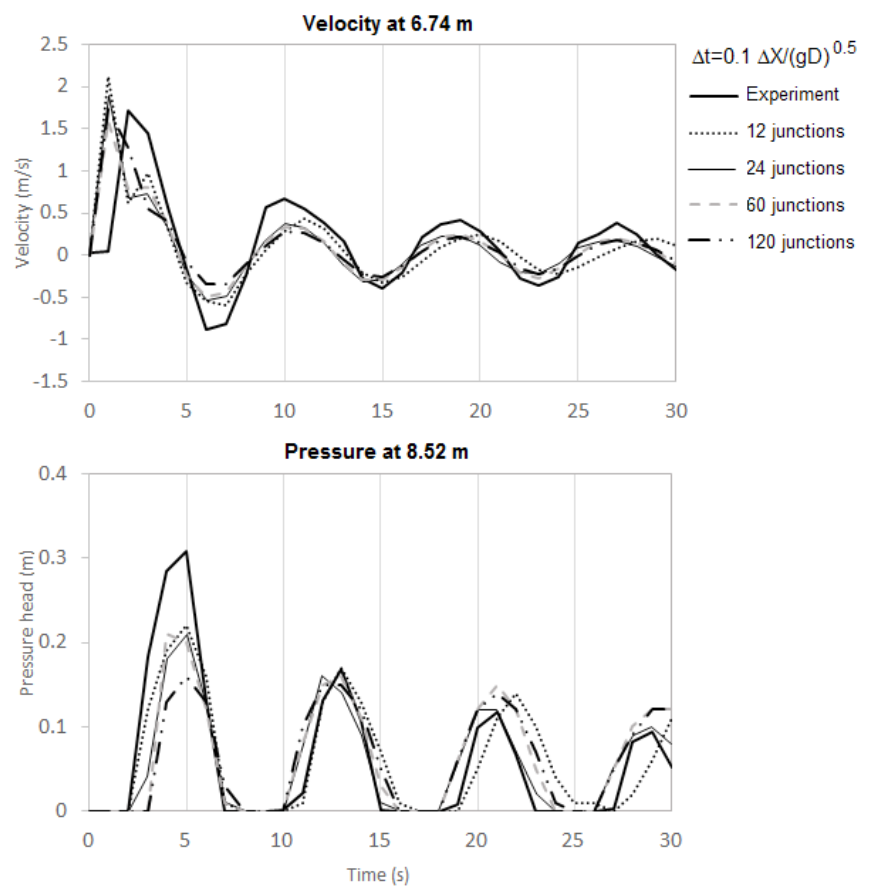

Figure 9 Experimental results (Aureli et al. 2015) and simulated results of water velocity at $X=6.74 \mathrm{~m}$ and pressure head at $X=8.52 \mathrm{~m}$ using SWMM and time step calculated by Equation 9 .

\section{Conclusions and Future Work}

The conditions that were simulated with SWMM in this work are not cases that are typically simulated, but rather represent conditions of rapid filling leading to flow pressurization. While the cases all involved reduced scale experimental investigations, the unsteady flow conditions represented were very complex. The traditional link-node representation used in SWMM is not able to capture the dynamics of rapid flow change, and the present work evaluated the model accuracy for different degrees of spatial discretization and time steps. Spatial discretization was realized by 
introducing dummy junctions, following Ridgway and Kumpula (2008), whereas routing time steps values were ensured by disabling the SWMM option for variable time steps.

The findings of the work may be summarized as follows:

1. The combination of discretization and adequate time step size has a major impact in the accuracy of the simulation. In general, better results were achieved when the time step sizes were reduced as the number of intermediate junctions increased. This was the case for the simulations representing the experiments involving a pipe filling bore presented by Vasconcelos (2005). In general, simulated surge tank water levels agreed more closely with experimental measurements than velocity results.

2. Simulations of a backward-moving pipe filling bore advancing over supercritical flow by Trajkovic et al. (1999) showed instabilities in pressure results, which were more severe when the time step was reduced. There was no clear trend linking discretization and time step size.

3. The comparison between the Aureli et al. (2015) experimental and SWMM simulation results followed in general the same patterns observed in the comparison involving the Vasconcelos (2005) experiments. It was particularly interesting to notice that the process of advance and retreat of water in the conduit (wetting and drying) was well represented in SWMM.

4. The recommendation by Roesner et al. (1988) for time step sizes to ensure the numerical stability of a SWMM model (Equation 7) was not adequate for all alternatives of internal discretization. In general, continuity errors were elevated, and there was poor agreement between modeling results and experimental observations. These errors were reduced when the time steps were reduced.

5. An alternative that was used which retained the simplicity of the Roesner et al. (1988) time step recommendation was to use only $10 \%$ of the time step size calculated by Equation 7. All simulations were performed with this reduced time step, and agreements were adequate for all intermediate junctions used in this study.

6. There were no obvious advantages in having larger numbers of intermediate junctions in terms of SWMM modeling accuracy. On the other hand, for conditions tested in this paper, the use of 10 to 12 intermediate junctions yielded simulation results that showed some time lag in the results. Depending on the length of the conduit and the degree of unsteadiness of the flow, the length of intermediate reaches $L$ that are recommended are in the range of 4 to 6 times the conduit diameter D.

One important point to be made is that this work has not systematically evaluated the impact of time step size and discretization to the stability of SWMM. EXTRAN solutions rely on successive approximations and use of a relaxation factor to update flows and head at junctions (Rossman 2017). Future studies will evaluate the effect of the relaxation factor, maximum number of trials and convergence tolerance in the accuracy of the solutions.

Also, further investigation will be directed towards the development of an automatic script that will implement an automatic discretization routine in a SWMM model link-node topology. Finally, alternative methods to define time step sizes (e.g. based on actual Courant number calculation) will be considered in future investigations.

\section{References}

Aureli, F., S.. Dazzi, A. Maranzoni and P. Mignosa. 2015. “Validation of Single- and Two-Equation Models for Transient Mixed Flows: A Laboratory Test Case" Journal of Hydraulic Research 53 (4): 440-51.

Bousso, S., M. Daynou and M. Fuamba. 2013. “Numerical Modeling of Mixed Flows in Storm Water Systems: Critical Review of Literature." Journal of Hydraulic Engineering 139 (4): 440-51.

Cunge, J. A. and M. Wegner. 1964. "Intégration numérique des équations d'écoulement de Barré de Saint-Venant par un schema implicite de différences finites: Application au cas d'une galerie tantôt en charge tantôt à surface libre." La Houille Blanche 1:33-9.

Gironas, J., L. A. Roesner and J. Davis. 2009. Storm Water Management Model Applications Manual. Washington, DC: U.S. Environmental Protection Agency. EPA/600/R-09/077.

Guo, Q. and C. C. S. Song. 1990. "Surging in Urban Storm Drainage Systems." Journal of Hydraulic Engineering 116 (1): 1523-37.

Guo, Q. and C. C. S. Song. 1991. "Dropshaft Hydrodynamics Under Transient Conditions." Journal of Hydraulic Engineering 117 (8): 1042-55.

Ridgway, K. and G. J. Kumpula. 2008. “Surge Modeling in Sewers Using Alternative Hydraulic Software Programs." Journal of Water Management Modeling R228-10.

Roesner, L. A., J. A. Aldrich and R. E. Dickinson. 1988. Storm Water Management Model User's Manual, Version 4: Addendum I, EXTRAN. Athens, GA: U.S. Environmental Protection Agency. EPA/600/3-88/001b (NTIS PB88236658/AS).

Rossman, L. A. 2006. Storm Water Management Model Quality Assurance Report: Dynamic Wave Flow Routing. Washington, DC: U.S. Environmental Protection Agency. EPA/600/R-06/097.

Rossman, L. A. 2017. Storm Water Management Model Reference Manual Volume II-Hydraulics. Washington, DC: U.S. Environmental Protection Agency. EPA/600/R-17/111.

Song, C. C. S., J. A. Cardle and K. S. Leung. 1983. "Transient MixedFlow Models for Storm Sewers." Journal of Hydraulic Engineering 109 (11): 1487-504. 
Toro, E. F. 2001. Shock-Capturing Methods for Free-Surface Shallow Flows. Chichester, NY: Wiley.

Trajkovic, B., M. Ivetic, F. Calomino and A. D'Ippolito. 1999. “Investigation of Transition from Free Surface to Pressurized Flow in a Circular Pipe." Water Science and Technology 39 (9): 105-12.

USEPA (U.S. Environmental Protection Agency). 2017. Storm Water Management Model (SWMM) ver. 5.0.12. Washington, DC: U.S. Environmental Protection Agency. https://www.epa.gov/water-research/storm-watermanagement-model-swmm

Vasconcelos, J. G. 2005. Dynamic Approach to the Description of Flow Regime Transition in Stormwater Systems. Ann Arbor, MI: University of Michigan. PhD dissertation.

Vasconcelos, J. G., P. R. Klaver and D. J. Lautenbach. 2015. “Flow Regime Transition Simulation Incorporating Entrapped Air Pocket Effects." Urban Water Journal 12 (6): 488-501.
Vasconcelos, J. G., S. J. Wright and P. L. Roe. 2006. "Improved Simulation of Flow Regime Transition in Sewers: Two-Component Pressure Approach." Journal of Hydraulic Engineering 132 (6): 553-62.

Wright, S. J., J. W. Lewis and J. G. Vasconcelos. 2011. “Physical Processes Resulting in Geysers in Rapidly Filling Storm-Water Tunnels." Journal of Irrigation and Drainage Engineering 137 (3): 199-202.

Wylie, E. B. and V. S. Streeter. 1993. Fluid Transients in Systems. Englewood Cliffs, NJ: Prentice Hall.

Zhou F., F. E. Hick and P. M. Steffler. 2002. "Transient Flow in a Rapidly Filling Horizontal Pipe Containing Trapped Air." Journal of Hydraulic Engineering 128 (6): 625-34. 